\title{
A Carbamazepine-induced Brugada-type Electrocardiographic Pattern in a Patient with Schizophrenia
}

\author{
Hisanobu Ota ${ }^{1}$, Yuichiro Kawamura ${ }^{2}$, Nobuyuki Sato ${ }^{2}$ and Naoyuki Hasebe ${ }^{2}$
}

\begin{abstract}
:
We report the case of a 61-year-old man with schizophrenia who was treated with carbamazepine, in whom electrocardiography showed transient Brugada-type ST elevation. He had been hospitalized our hospital's Department of Psychiatry and had been diagnosed with pneumonia. On the following day, electrocardiography showed coved-type ST elevation in the right precordial leads and a blood examination revealed that the patient's carbamazepine concentration was at the upper limit of the standard range, as well as hypothyroidism. The patient's electrocardiogram normalized after the withdrawal of carbamazepine. We demonstrated that the patient's carbamazepine concentration-and not hypothyroidism-was associated with the serial electrocardiographic changes by monitoring the patient's blood concentration of carbamazepine and his thyroid function.
\end{abstract}

Key words: Brugada-type electrocardiography, carbamazepine, schizophrenia, antiepileptic drug, sodium channel

(Intern Med 56: 3047-3050, 2017)

(DOI: 10.2169/internalmedicine.8875-17)

\section{Introduction}

Brugada syndrome is a cardiac channelopathy that is characterized by coved-type or saddleback-type ST segment elevation in the right precordial leads on electrocardiography. It has the potential to cause fatal ventricular arrhythmias (1). On the other hand, it has been reported that the mortality rate is elevated in patients with schizophrenia in comparison to the general population. Ischemic heart disease and drug-induced QTc interval prolongation are considered to be possible mechanisms (2-4). Furthermore, schizophrenia is reported to be associated with an increased risk of sudden cardiac death and a higher prevalence of Brugada-type electrocardiography findings $(5,6)$. In addition, it was recently reported that antidepressants and other psychoactive drugs may provoke a Brugada-type electrocardiographic pattern (7). We encountered a case of schizophrenia in a patient who was treated with carbamazepine who presented with a transient coved-type Brugada electrocardiographic pattern and hypothyroidism. We herein discuss the possible mechanisms based on the findings of our case.

\section{Case Report}

The patient was a 61-year-old man with schizophrenia who had been hospitalized in our hospital's Department of Psychiatry since December 2014. The patient's medications (all administered orally) included: carbamazepine $(200 \mathrm{mg}$ ) and valproic acid (200 $\mathrm{mg}$ ) twice a day, and olanzapine (10 $\mathrm{mg}$ ), flunitrazepam $(2 \mathrm{mg})$ and quazepam $(15 \mathrm{mg})$ at bedtime. Thiamazole had also been prescribed since another physician had diagnosed the patient with hyperthyroidism 35 years previously. During hospitalization, the dose of the thiamazole was controlled by a psychiatrist because thiamazole-induced hypothyroidism emerged.

At the end of November, 2015, he developed fever and productive cough. He was diagnosed with pneumonia by

${ }^{1}$ Department of Cardiology, Hakodate Watanabe Hospital, Japan and ${ }^{2}$ Division of Cardiology, Nephrology, Pulmonology and Neurology, Department of Internal Medicine, Asahikawa Medical University, Japan

Received for publication January 16, 2017; Accepted for publication January 29, 2017

Correspondence to Dr. Hisanobu Ota, hisa128@asahikawa-med.ac.jp 


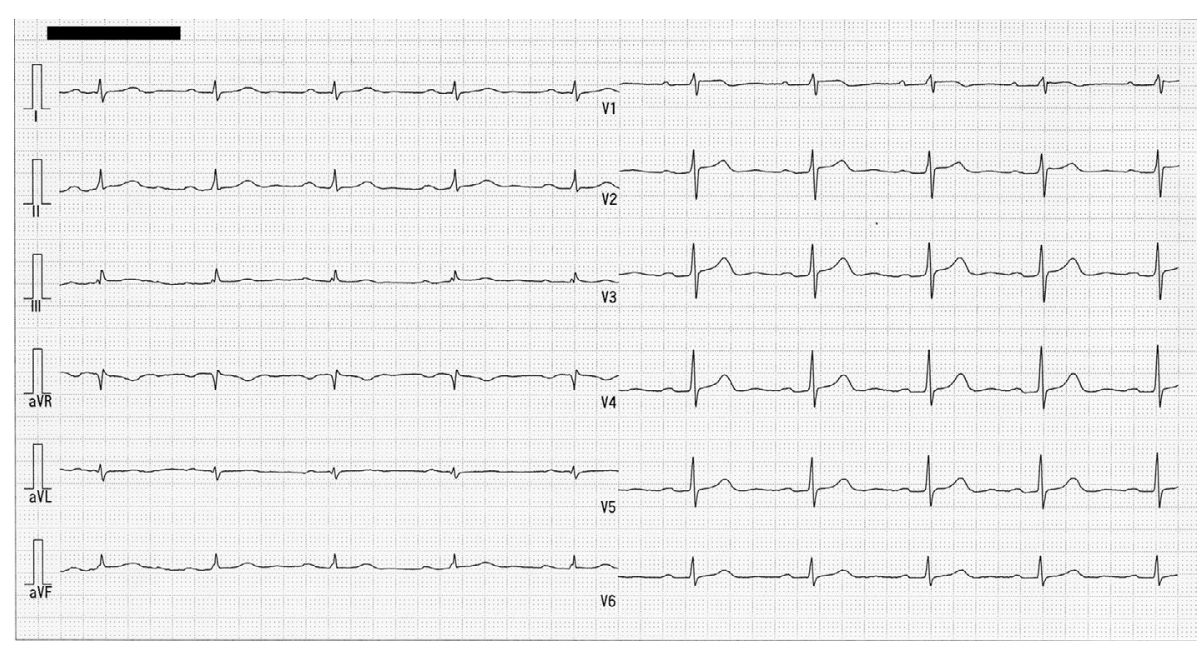

Figure 1. Electrocardiography was performed before the episode. No ST segment elevation was observed in the right precordial leads.

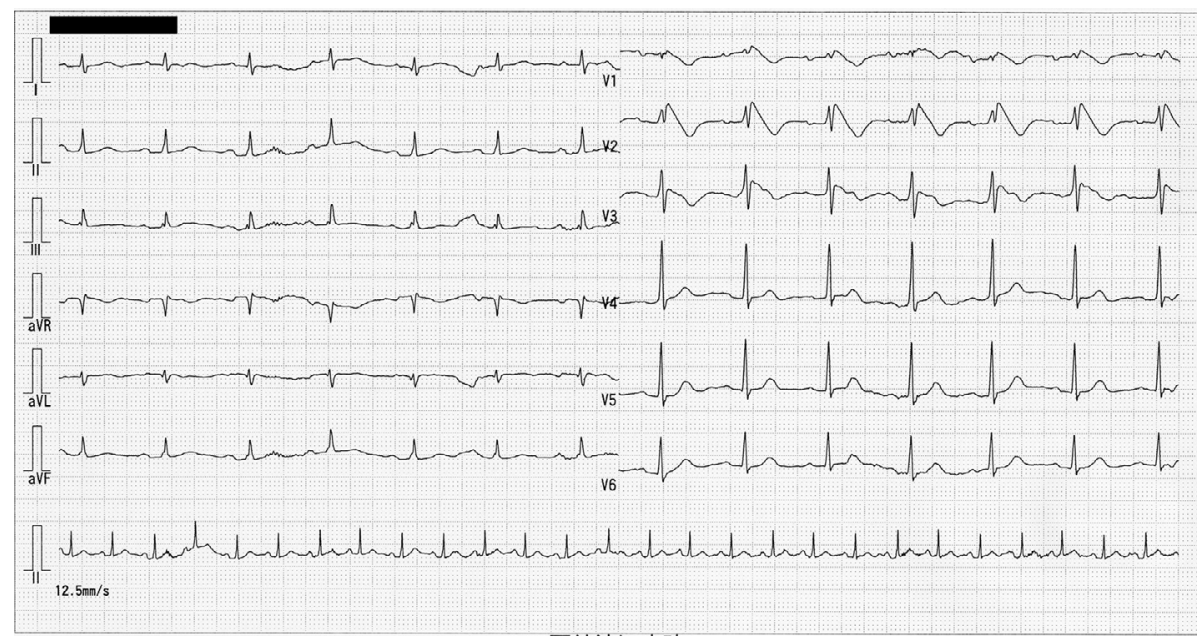

Figure 2. A Brugada-type electrocardiographic pattern can be seen in the right precordial leads.

chest computed tomography, and was treated with intravenous antibiotics. Since his blood examination data revealed abnormal creatinine phosphokinase elevation on the following day, electrocardiography was performed. During his hospitalization in the Department of Psychiatry, the patient had undergone electrocardiography at least once a month, and no Brugada-type waveforms had been seen (Fig. 1). On the electrocardiogram that was obtained after the diagnosis of pneumonia, however, coved-type ST segment elevation was observed in leads V1-3 (Fig. 2). He was therefore transferred to our department for a close examination and treatment.

A physical examination revealed mild wheezing, edema in the lower extremities, and ocular proptosis. The patient's blood pressure was $127 / 72 \mathrm{mmHg}$, his heart rate was 90 beats per minute, and his body temperature was $37.4^{\circ} \mathrm{C}$. He had no previous episodes of syncope and no family history of sudden cardiac death. The patient's serum creatinine phosphokinase level was elevated $(1,618 \mathrm{U} / \mathrm{L})$, while his creatine kinase (CK)-MB level showed slight elevation (24.3 $\mathrm{ng} / \mathrm{mL}$ ). The patient's serum potassium level was $3.4 \mathrm{mEq} /$ $\mathrm{L}$, and his C-reactive protein level was $28.46 \mathrm{mg} / \mathrm{dL}$. Hypothyroidism was reconfirmed based on a thyroid stimulating hormone (TSH) level of $23.49 \mu \mathrm{IU} / \mathrm{mL}$ and free-T4 level of $<0.10 \mathrm{ng} / \mathrm{dL}$. It was noteworthy that the patient's blood concentration of carbamazepine reached the upper limit of the standard range $(11.9 \mu \mathrm{g} / \mathrm{mL})$, while the blood concentration of valproic acid was relatively lower than the standard range (43 $\mu \mathrm{g} / \mathrm{mL})$.

We withdrew the administration of carbamazepine, valproic acid, and thiamazole. Thereafter, the coved-type ST segment elevation improved in proportion to the decrease in the blood concentration of carbamazepine. Although the thyroid function eventually normalized, it was not correlated with the serial electrocardiographic changes. Furthermore, the patient's body temperature (whether the patient was febrile or not) was not associated with the electrocardiographic changes (Fig. 3). 


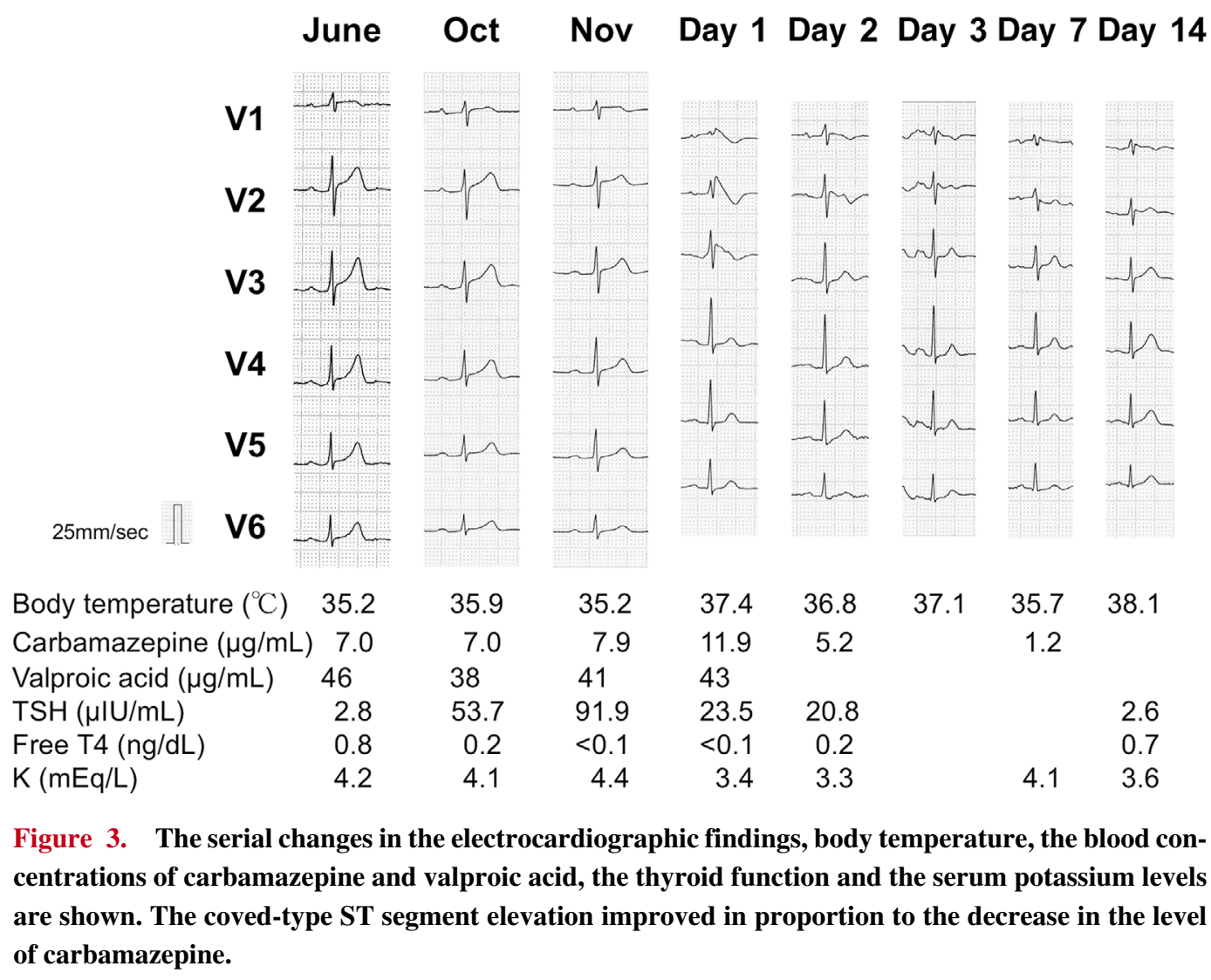

\section{Discussion}

Carbamazepine is an antiepileptic drug that blocks the activity of the sodium channels. It is also used to treat aggressive symptoms in schizophrenia. There are few reports of carbamazepine-induced Brugada-type patterns on electrocardiography. The only published case involved a patient whose blood concentration of carbamazepine reached $338 \mu \mathrm{mol} / \mathrm{L}$ $(80 \mu \mathrm{g} / \mathrm{mL})$ after suffering poisoning due to the ingestion of $32 \mathrm{~g}$ of slow-release carbamazepine (8). In our case, the Brugada-type electrocardiographic pattern was correlated with the blood concentration of carbamazepine, despite the patient receiving a usual dosage. Valproic acid is also known to have a sodium channel blocking effect. However, the patient's blood concentration of valproic acid was not elevated during hospitalization. Unfortunately, we could not perform a genetic analysis to investigate channelopathy.

Patients with schizophrenia are reported to be at increased risk of sudden cardiac death (5). Furthermore, Blom et al. reported that a Brugada-type electrocardiographic pattern is highly prevalent in patients with schizophrenia (6). They analyzed the electrocardiograms of a cohort of 275 patients with schizophrenia and compared it to an age-matched nonschizophrenic control group $(\mathrm{n}=179)$ and a control group composed of non-schizophrenic individuals who were 20 years older $(n=1,168)$ and found that Brugada-type electrocardiographic patterns were significantly more prevalent in patients with schizophrenia cohort (11.6\%) than they were in the age-matched cohort (1.1\%) and the cohort that was 20 years older (2.4\%). However, at the time of writing, there is no evidence of a tight association between schizophrenia and sodium channelopathy.

In our case, hypothyroidism was observed as an effect of thiamazole. Several cases of hypothyroidism associated with Brugada-type electrocardiographic patterns have been reported previously $(9,10)$. It is possible that the Brugadatype electrocardiographic patterns in these patients were caused by a reduction in the $I_{N a}$ and sympathetic nervous system activity in patients with a low thyroid function; however, there is no clear evidence. In our case, there was no close association between the thyroid function and the electrocardiographic morphology, as evidenced by the fact that the patient's electrocardiograms were normal before this episode, in spite of his hypothyroidism. However, it is possible that the patient's hypothyroidism might have played a complementary role in causing coved-type ST elevation, by enhancing the sodium channel blockade of carbamazepine.

Recently, Ishizue et al. reported that polytherapy with sodium channel-blocking antiepileptic drugs may be associated with Brugada-type ST elevation and J-wave-like electrocardiographic abnormality in the patients with epilepsy (11). They mentioned that the use of phenytoin and polytherapy with sodium channel-blocking antiepileptic drugs was more frequent among the group of patients who were Brugadatype ST elevation-positive than it was among the group of patients who were Brugada type ST elevation-negative. However, the rate of carbamazepine administration did not differ to a statistically significant extent. In our case, only the carbamazepine concentration was tightly associated with 
the electrocardiographic changes in Brugada-type ST elevation.

In our case, the patient's serum carbamazepine concentration had been stable until the patient was diagnosed with pneumonia. This may be educational because we have to carefully monitor patients with schizophrenia or epilepsy for electrocardiographic changes, even when the carbamazepine concentration is within the normal range. When patients who are treated with carbamazepine show deterioration in their general condition, we remember to perform electrocardiography. If coved-type ST elevation is found in lead V1 or 2 , we should monitor the serum concentration of carbamazepine and consider the indications for withdrawal. In order to prepare for such situations, electrocardiography should be periodically performed when the patient is in a stable condition.

In conclusion, we reported the case of a patient with schizophrenia who was treated with carbamazepine who presented with a transient coved-type Brugada electrocardiographic pattern and hypothyroidism. During the course of the patient's hospitalization, the monitoring of the blood concentration of carbamazepine and the thyroid function revealed that the level of carbamazepine was deeply associated with the serial electrocardiographic changes.

\section{The authors state that they have no Conflict of Interest (COI).}

\section{Acknowledgement}

We would like to express our gratitude to Tadashi Hasegawa, Kiyoshi Mizuseki, Katsuyoshi Sakyo, Akihiro Mikami, Masahiko Mikuni and the staff at Hakodate Watanabe Hospital for helpful suggestions and their support.

\section{References}

1. Priori SG, Wilde AA, Horie M, et al. HRS/EHRA/APHRS expert consensus statement on the diagnosis and management of patients with inherited primary arrhythmia syndromes: document endorsed by HRS, EHRA, and APHRS in May 2013 and by ACCF, AHA, PACES, and AEPC in June 2013. Heart Rhythm 10: 1932-1963, 2013.

2. Reilly JG, Ayis SA, Ferrier IN, Jones SJ, Thomas SH. QTcinterval abnormalities and psychotropic drug therapy in psychiatric patients. Lancet 355: 1048-1052, 2000.

3. Saha S, Chant D, McGrath J. A systematic review of mortality in schizophrenia: is the differential mortality gap worsening over time? Arch Gen Psychiatry 64: 1123-1131, 2007.

4. Roden DM. The Brugada ECG and schizophrenia. Circ Arrhythm Electrophysiol 7: 365-367, 2014.

5. Ifteni P, Correll CU, Burtea V, Kane JM, Manu P. Sudden unexpected death in schizophrenia: autopsy findings in psychiatric inpatients. Schizophr Res 155: 72-76, 2014.

6. Blom MT, Cohen D, Seldenrijk A, et al. Brugada syndrome ECG is highly prevalent in schizophrenia. Circ Arrhythm Electrophysiol 7: 384-391, 2014.

7. Postema PG, Wolpert C, Amin AS, et al. Drugs and Brugada syndrome patients: review of the literature, recommendations, and an up-to-date website (www.brugadadrugs.org). Heart Rhythm 6: 1335-1341, 2009.

8. Megarbane B, Leprince P, Deye N, et al. Extracorporeal life support in a case of acute carbamazepine poisoning with lifethreatening refractory myocardial failure. Intensive Care Med 32: 1409-1413, 2006.

9. Kitahara A, Hirai R, Matsui Y, Ikeda Y, Nakamura H. A case of hypothyroidism with Brugada electrocardiographic waveforms. Endocr J 55: 589-594, 2008.

10. Taira K, Fujino A, Watanabe T, Ogyu A, Ashikawa K, Shimizu W. Brugada-type electrocardiogram in a patient with hypothyroidism. J Cardiol Cases 2: e147-e150, 2010.

11. Ishizue N, Niwano S, Saito M, et al. Polytherapy with sodium channel-blocking antiepileptic drugs is associated with arrhythmogenic ST-T abnormality in patients with epilepsy. Seizure 40: 8187, 2016.

The Internal Medicine is an Open Access article distributed under the Creative Commons Attribution-NonCommercial-NoDerivatives 4.0 International License. To view the details of this license, please visit (https://creativecommons.org/licenses/ by-nc-nd/4.0/).

(C) 2017 The Japanese Society of Internal Medicine Intern Med 56: 3047-3050, 2017 